\title{
Topology and size optimization of truss
}

\author{
Naresh D. Sonwane \\ Department of Applied Mechanics \\ Govt. College of Engineering, Amravati \\ Dr. K. N. Kadam \\ Faculty of Applied Mechanics Department \\ Govt. College of Engineering, Amravati
}

\begin{abstract}
The main purpose of the structural optimization is to reduce the manufacturing cost of the structure corresponding to material requirement in present era. However, economic design should not deviate from engineering restrictions, specification and laws. In order to increase the optimization speed and decrease the solution time of the problem, an efficient computer tool is used and analysis methods has been employed leading to reduction of the time required for computing of the objective function. HyperWork is known for handling global optimization problems when many local optima are present in a non-continuous system. The objective of present study is the minimization of mass with optional stress and displacement constraints. Stress or displacement constraints applied to stand a typical optimization problem will utilize and size capabilities. The first phase is that of the structure, containing all implications and capabilities in the static analysis of such problem. The second phase applies to that of structural optimization, more specifically the optimization of size and topology.
\end{abstract}

Keywords - Optimization, Size, Topology etc.

\section{INTRODUCTION}

Complex structures become difficult to optimize due to increase in variable interactions. The formalized subject of structural optimization became intensively researched in the latter half of the 20th century, largely due to developments in numerical techniques and efficient and powerful computational hardware and the needs of the aerospace, civil engineering structures, biotechnology and automotive industry. This coincided largely with the development of numerical analysis methods, most prominently the Finite Element Method (FEM). Simply stated, the aim of structural optimization is to find the layout of structural material with specified properties that provides optimal structural performance, while satisfying a number of requirements of the problem.

The type of structural optimization can be categorized in terms of the structural property to be optimized, sizing, shape or topology:

- Sizing optimization considers the cross sectional dimensions as design variables. An area of members has to be variable.

- Shape optimization considers the geometrical variables related to the shape of the structure. The topology remains constant. Nodal coordinates mainly has to be variable.

- Topology optimization involves defining the optimal distribution of material, often from a given initial distribution, called 'ground structure' in truss problems, or a bulk of material in continuum structures.

The objective of the structural optimization is the minimization of mass with optional stress and displacement constraints. Stress or displacement constraints applied to stand a typical optimization problem will utilize shape, topology and size capabilities. The first definition is that of the structure, containing all implications and capabilities in the static analysis of such problem. The second definition applies to that of structural optimization, more specifically the optimization of size and topology.

All elements are associated with a material defined by a minimum of two values: modulus of elasticity (E) and Poisson's ratio (n). These values define the element's behaviour under static linear elastic loading conditions. Values used only for the optimization process include the element's yield strength, and unit weight or mass density. These values are used for stress limit comparison and structural mass, respectively.

The method uses the following criteria to prefer one solution on another:

- Any feasible solution is preferred to any infeasible solution.

- Among two feasible solutions, the one having better objective function value preferred.

- Among two infeasible solutions, the one having smaller constraints violation is preferred.

Size Optimization Based on Model Geometry in this method, the linkage of an FE model and a geometry model is maintained. In this case the parameters of the geometry model, such as thickness, the dimensions of the cross 
section, etc., are the design variables. The geometry model has to be fully parametric. Each parameter modification of the geometry model creates a new design. The design variables are swept over a range of specified values and each design is analyzed and the results of the analysis are compared to impose behavioural constraints, such as stress, etc. A design is termed as a feasible design, if it satisfies all the imposed constraints (constraints on the maximum stress, etc.) on it. Otherwise, the design is termed as infeasible design.

The topology optimization of truss structures is a problem of determining the existence of the member of the truss between two nodes, such that the kinematic stability of the structure is preserved in the final design. A truss satisfying the kinematic stability condition is called a structure, and, otherwise, it is referred to as a mechanism. One of the ways is to check the stability of structure by using degree of freedom. Categorization of type of structure optimization of discrete and continuum structure is as shown in fig 1 . Six types of problems can be distinguished.

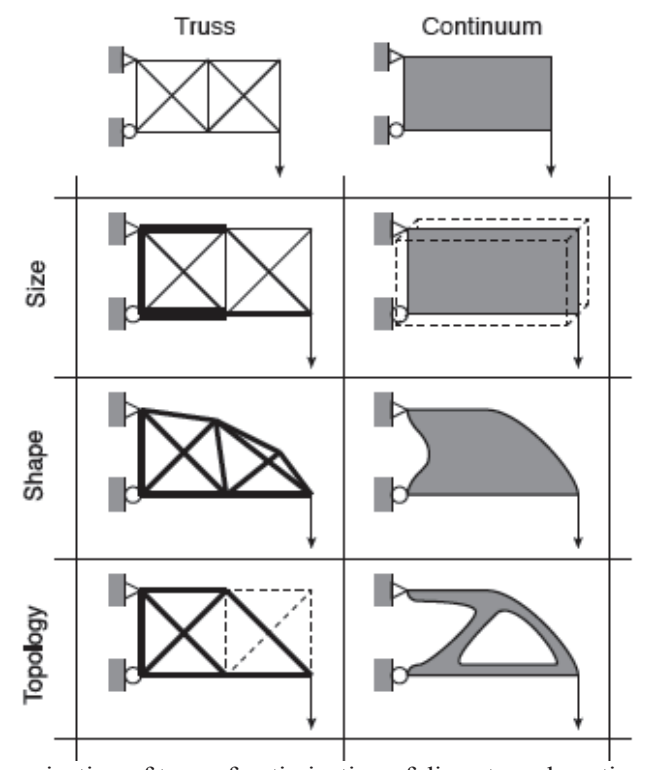

Figure 1: Categorization of type of optimization of discrete and continuum structures.

\section{II.ILLUSTRATIVE EXAMPLE}

The feasibility and effectiveness of the propose method are illustrated by two examples. These examples are often used to demonstrate the procedure used in optimization. In this paper size and topology is considered.

The first example is the case of a 11 member 6 noded 2D benchmark truss and it's optimizes topology [1]. Size optimization is further process over topologically best design truss having structural stability. Variation of area of member in respective iteration to optimize the axial stress and displacement capability under given set of constrains for minimize structural weight. Comparison of weight of individual member after size optimization is as shown in fig 2. 


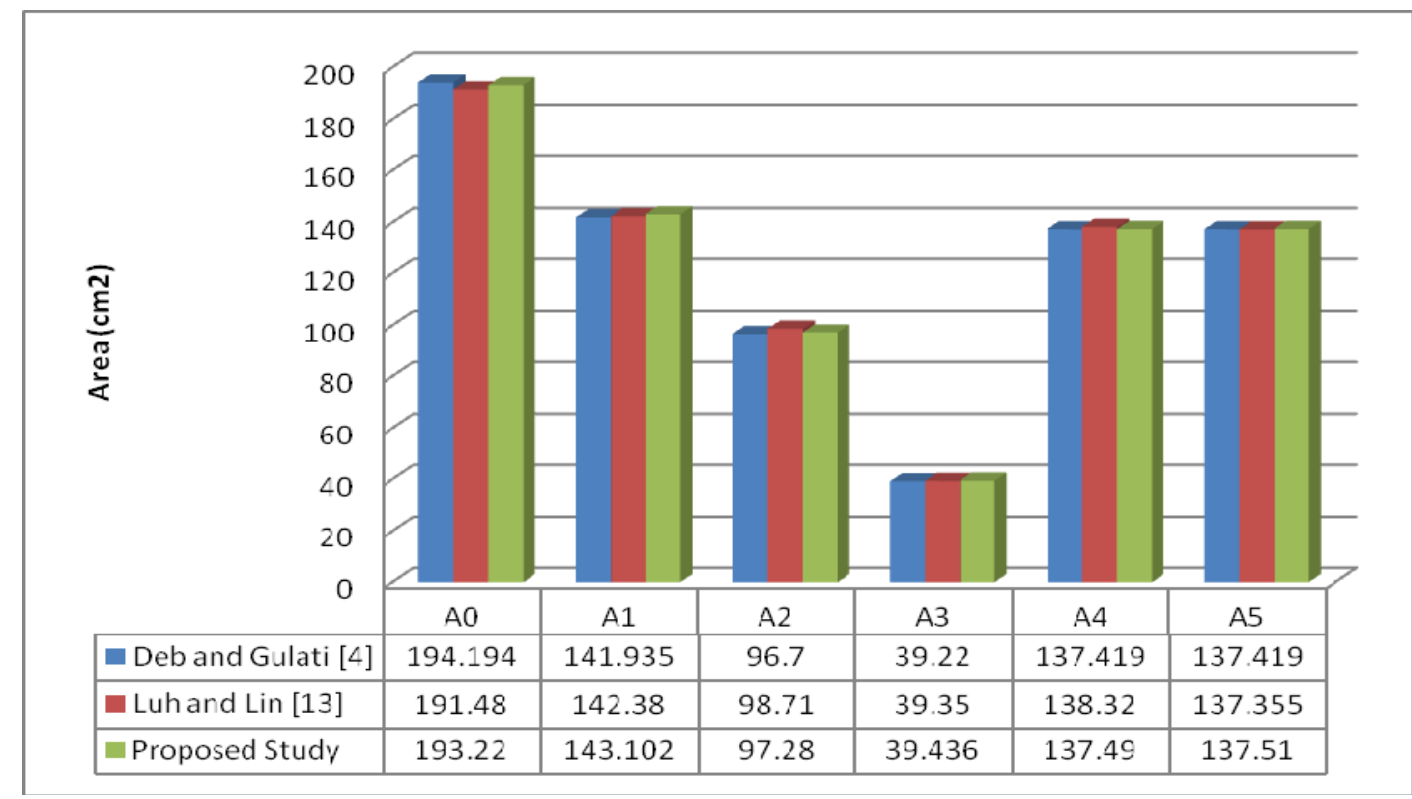

Figure 2: Comparison of Weight of Members

Final result of optimised weigh obtained by this study and result obtained by Luh and Lin are comparing in Table 1. It shows that after optimization weight equal to $35.74 \%$ to initial weight of structure. It also provides weight of truss after both topology and size optimization by Luh and Lin.

\begin{tabular}{|l|l|l|l|}
\hline \multicolumn{2}{|c}{ Table 1: Comparisons of Weights $(\mathrm{kg})$} \\
\hline Proposed study & 6262.67 & $\begin{array}{l}\text { Topology } \\
\text { Optimization }\end{array}$ & $\begin{array}{l}\text { Size }+ \text { Topology } \\
\text { Optimization }\end{array}$ \\
\hline Luh and Lin & 6260.26 & 3550.5 & 2235.54 \\
\hline
\end{tabular}

Displacement obtain after static analysis of benchmark truss is $35.5 \mathrm{~mm}$ and displacement of final design obtained by topology and size optimization is $50.69 \mathrm{~mm}$, as shown in Table 2, this values of displacement satisfy the constrains of displacement taken in the problem initially [5].

Table 2: Comparisons of Weights $(\mathrm{kg})$

\begin{tabular}{|l|l|l|l|}
\hline Parameter & Benchmark truss & $\begin{array}{l}\text { Topology } \\
\text { optimization }\end{array}$ & $\begin{array}{l}\text { size }+ \text { topology } \\
\text { optimization }\end{array}$ \\
\hline Displacement in $\mathrm{mm}$ & 35.5 & 42.3 & 50.69 \\
\hline
\end{tabular}

The second example is the case of a 15 bar 6 noded ground structure truss as shown in figure 3, having similar property to previous 11 member 6 noded truss. Topology and size optimization carried out of this truss. 


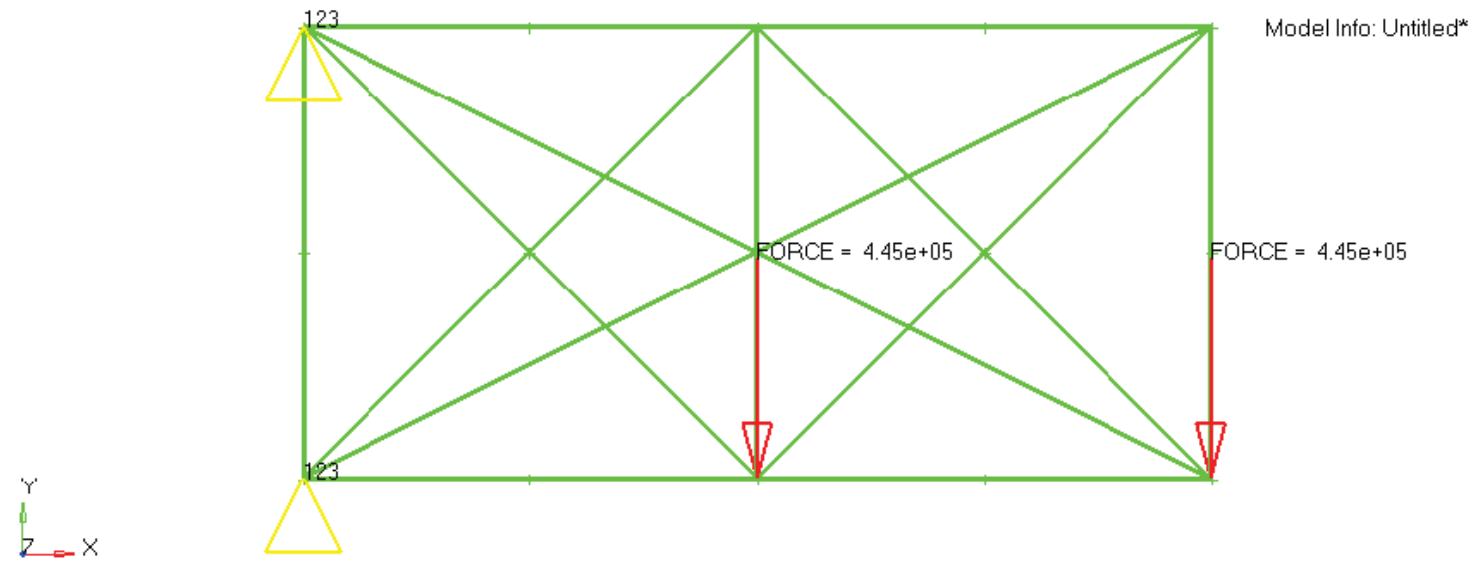

Figure 3: 15 bar 6 Noded Ground Structure

Ground structures of truss consisting every possibility of present of member between any two nodes this represent all nodes are joint each other with one member. In this case topology of truss has change on the basis of deflection allowed. Topology and respective deflection are shown in fig 4 and fig 5.

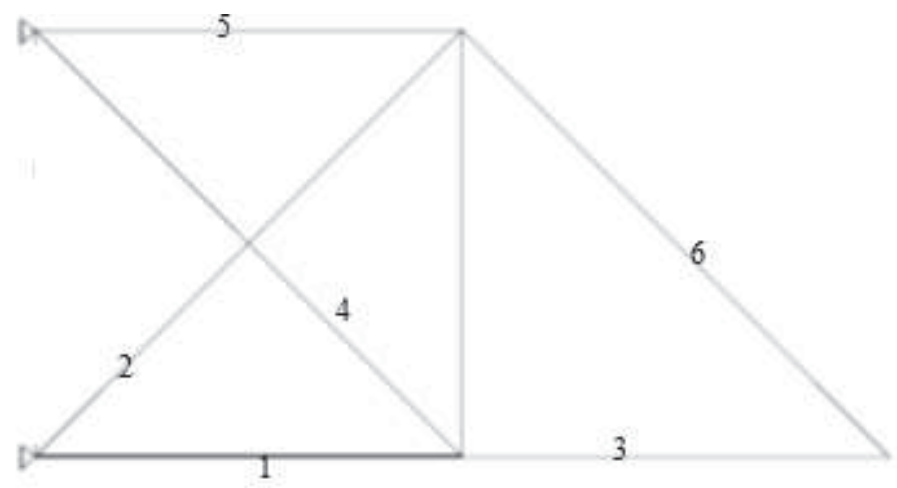

Figure 4: Topology of Truss (Max Displacement 50.8mm)

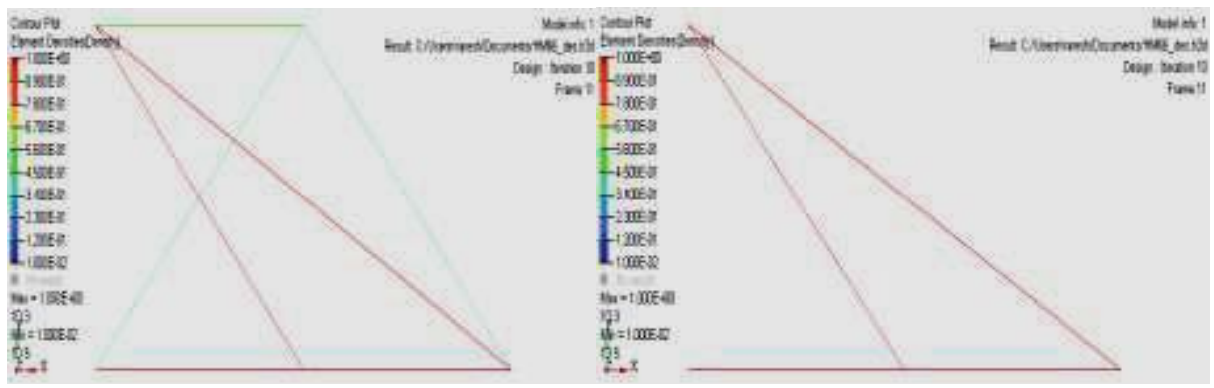

Figure 5: Topology of Truss (Max Displacement 70mm and 85mm)

Size optimization is further process over topologically best design truss having structural stability. Variation of area of member in respective iteration to optimize the axial stress and displacement capability under given set of constrains for minimize structural weight. Comparison of weight of individual member after size optimization is as shown in figure 6. 


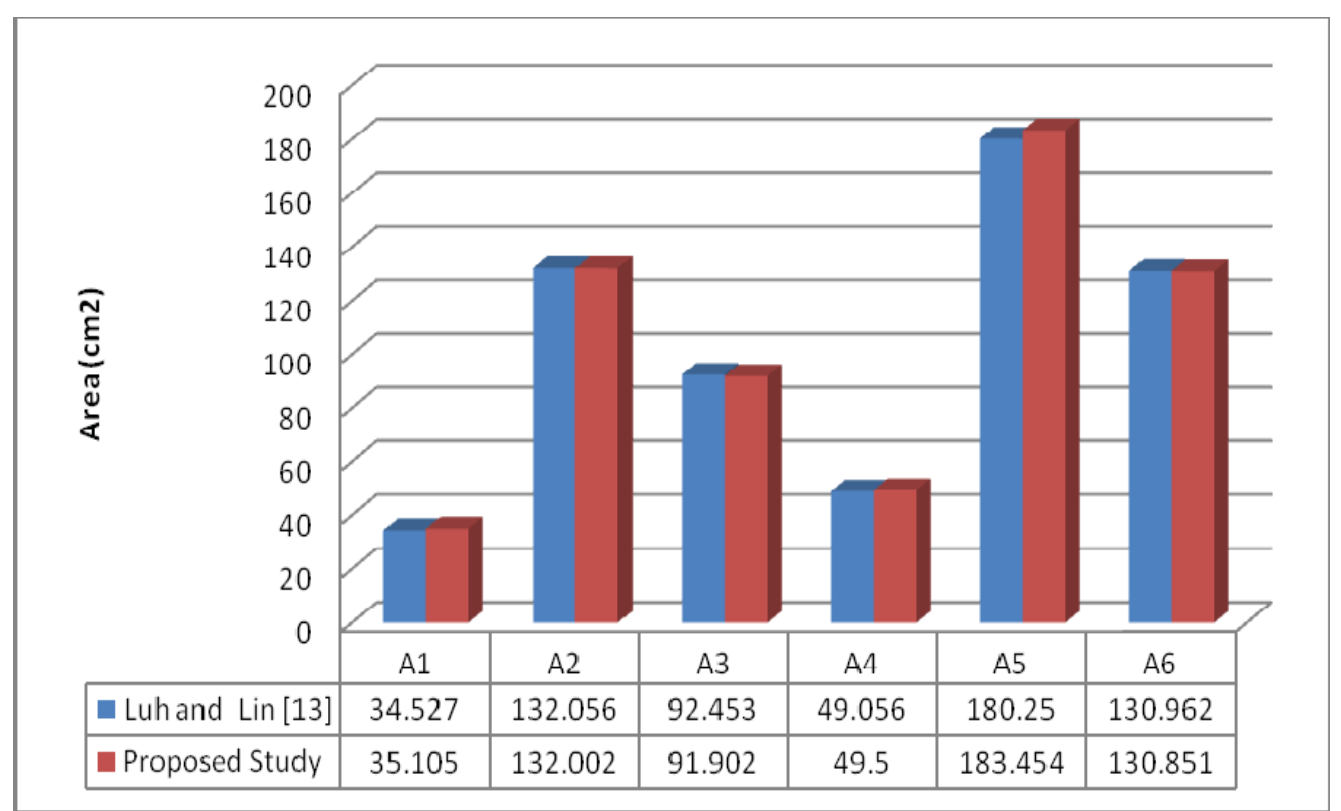

Figure 6: Comparison of Weight of Members

The third example is the case of a 25 bar 10 noded space Truss shown in fig 7 having material property similar to 11 member 6 noded truss [9]. The structure is imposed by two load cases listed in Table 3, all the nodal displacement constrains to be $\leq 8.89 \mathrm{~mm}$. The structure has required to symmetry with respect to $\mathrm{x}-\mathrm{y}$ plane and $\mathrm{z}-\mathrm{y}$ plane. Figure 7 shows A. side and B. top view of truss.

\begin{tabular}{|c|c|c|c|c|}
\hline \multicolumn{5}{|c|}{ Table 3: Nodes and Respective Load Case } \\
\hline Load Case & Node & $\mathbf{F}_{\mathbf{X}}(\mathbf{k N})$ & $\mathbf{F}_{\mathbf{Y}}(\mathbf{k N})$ & $\mathbf{F}_{\mathbf{Z}}(\mathbf{k N})$ \\
\hline 1 & 1 & 4.45 & -22.25 & 44.5 \\
\hline 1 & 2 & 0 & -22.25 & 44.5 \\
\hline 1 & 3 & 2.225 & 0 & 0 \\
\hline 2 & 6 & 2.225 & 0 & 0 \\
\hline 2 & 1 & 0 & -22.25 & 89 \\
\hline
\end{tabular}




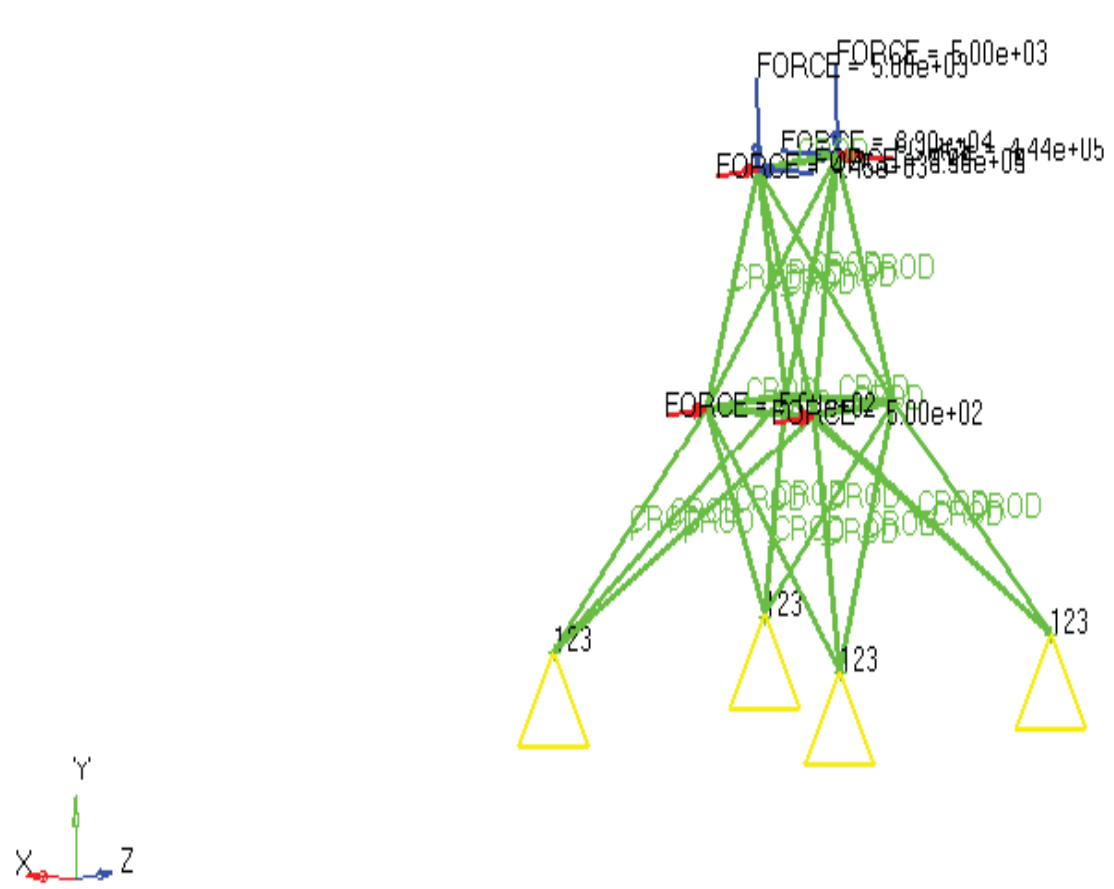

Model Info: Untitled*

Figure 7: A. Side View of Tress and B. Top View of Truss

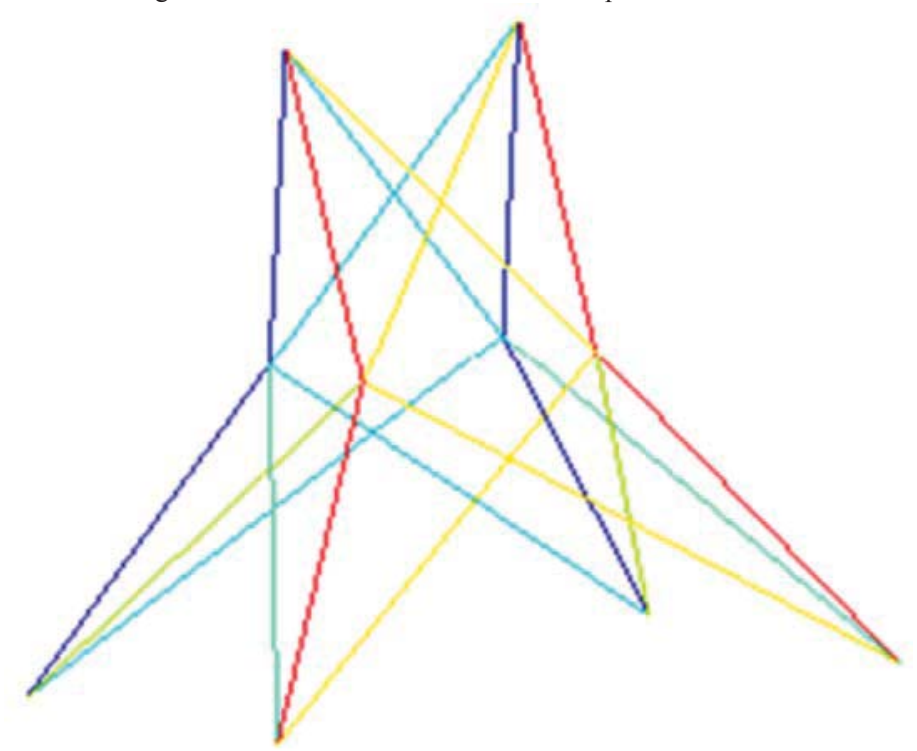

Figure 8: Topology of space truss

Optimize topology of space truss is as shown in fig 8 . Convergence histories of optimization process are as shown in fig 9 and fig 10 converged results at $20^{\text {th }}$ iteration. The members are divided in five groups and distinguished based on areas of members. Weight reduced from $830 \mathrm{~kg}$ to $215 \mathrm{~kg}$. After topology and size optimization optimize displacement up to $8.89 \mathrm{~mm}$ at every node. 


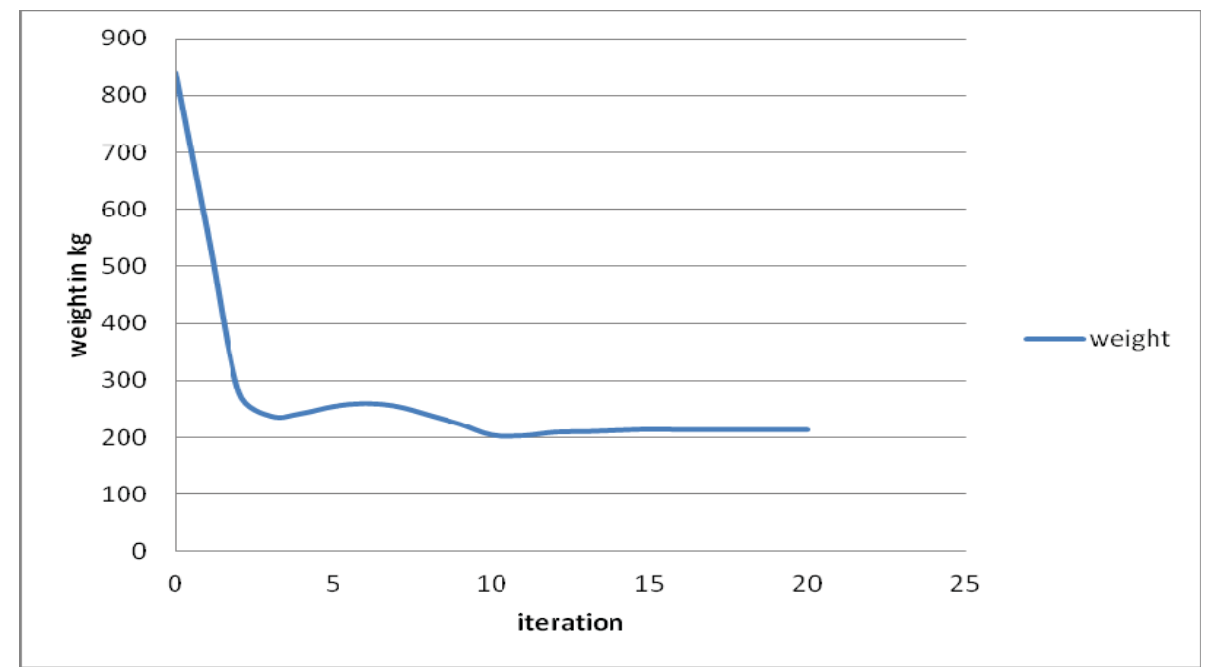

Figure 9: Convergence History of Weigh

Convergence of displacement is shown in fig 10. Displacement is converged up to $20^{\text {th }}$ iteration respected to optimized weight. Areas of respective members are shown in Table 4.

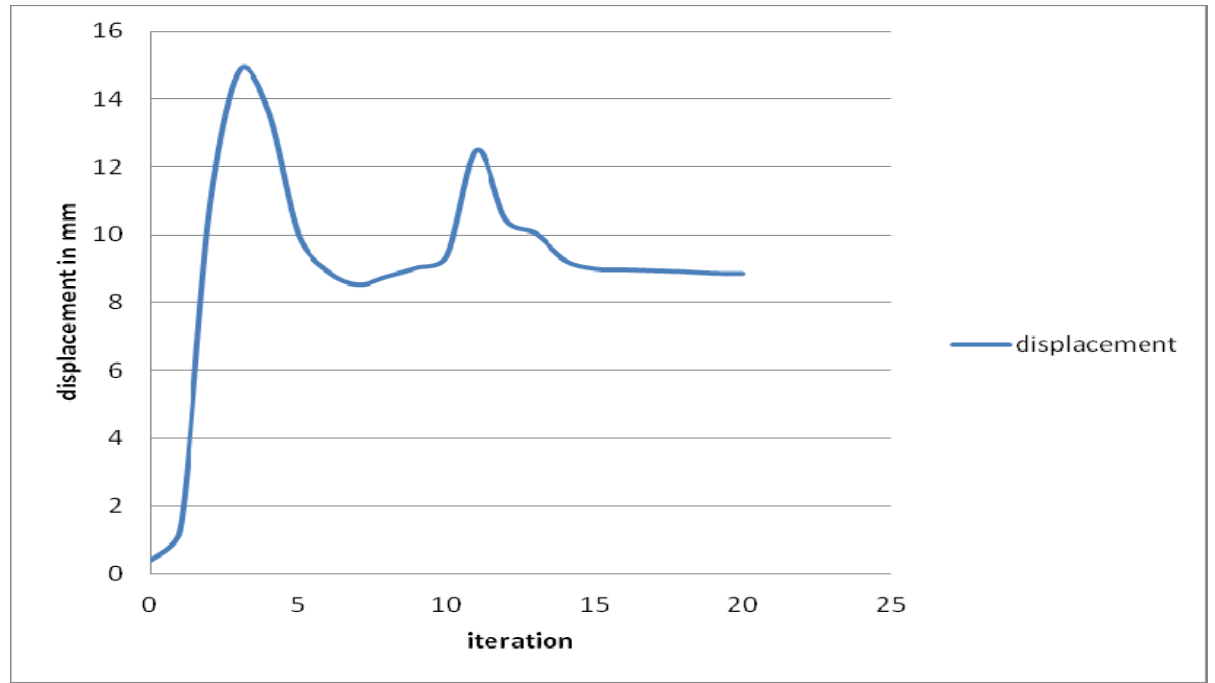

Figure 10 Converge History of Displacement

\begin{tabular}{|c|c|c|}
\hline \multicolumn{2}{c|}{} & Table 4: Member Area Variation without topology optimization \\
\hline Variable & Members & Area of Members $\mathbf{c m}^{2}$ \\
\hline A1 & 2 to 5 & 0.645 \\
\hline A2 & 6 to 9 & 22.581 \\
\hline A3 & 14 to 17 & 5.161 \\
\hline A4 & 18 to 21 & 16.129 \\
\hline A5 & 22 to 25 & 16.129 \\
\hline
\end{tabular}

III.CONCLUSION

In the present study, an optimization technique for indeterminate truss has been studied using HyperWork software. Proposed approach uses element densities and area of the member as design variable and nodal displacements and stresses are constraints. The following conclusions are made.

1. Objective function for minimizing the weight is depends upon various design variable parameters but predominantly contribute are number of members, area of members, nodal co-ordinates. 
2. Number of iteration to obtain the results is less it means convergence of result is achieved in less computational time for less number of member containing trusses.

3. Linear static result produce by tool is similar to the results obtained by analytical method.

4. Topology optimization results in similar topology produced in literature it means method and procedures are working properly.

5. Topology Optimization gives weight reduction in the range of 50 to 60 percentages of initial structure again size optimization gives weight reduction up-to $20 \%$ weight of topological optimized truss. Final truss weight is nearly 40 percentage weight of initial truss.

The present work is a good contribution to use the structures to its maximum capacity and to enhance nation economy by reduced material consumptions.

\section{REFERENCES}

[1] Naresh D. Sonwane, K.N. Kadam / International Journal of Engineering Research (IJER) ISSN:2319-6890 "Topology Optimization of Truss", Volume No.5 Issue: Special 3, 2016, pp: 621-624.

[2] Practical aspect of structural optimization, 2nd Edition Released 06/2015 Altair University.

[3] Practical aspect of finite element simulation, 3rd Edition Released 05/2015 Altair University.

[4] Luh, G.C. and Lin, C.Y. “optimal design of truss structure using ant algorithm”,Struct. Multidiscip. Optim. 36. Pp. 365-379 2008.

[5] Deb, K. and Gulati, K., Design of truss-structures for minimum weight using genetic algorithms" Kanpur Genetic Algorithms Laboratory (KanGAL), Depart- ment of Mechanical Engineering, Indian Institute of Technology, Kanpur, pp. 281-297.

[6] D. Wang, W.H. Zhang, J.S. Jiang, "Truss shape optimization with multiple displacement constraints", Comput. Methods Appl. Mech. Engrg. 191 (2002) 3597-3612.

[7] Ismail Farajpour, "A coordinate descent based method for geometry optimization of trusses", Advances in Engineering Software 42 (2011) $64-75$.

[8] A. Faramarzi, M.H. Afshar, "Application of cellular automata to size and topology optimization of truss structures", Scientia Iranica A (2012) 19 (3), 373-380.

[9] Forest Flager, Grant Soremekun, Akshay Adya, Kristina Shea, John Haymaker, Martin Fischer, "Fully Constrained Design: A general and scalable method for discrete member sizing optimization of steel truss structures", Computers and Structures 140 (2014) 55-65 